\title{
Los límites del concepto de frontera en distintas teorías antropológicas posmodernas
}

\author{
THE LIMITS OF THE CONCEPT FRONTIER IN DIFFERENT POSTMODERN ANTHROPOLOGICAL THEORIES
}

Dra. Isabel G. Gamero Cabrera (ig.gamero@filos.ucm.es) Instituto de Investigaciones Feministas, Universidad Complutense de Madrid (Madrid, España)

\begin{abstract}
The main interest of this paper is to understand how different theories have influence in the anthropological study of boundaries spaces. First, I will refer to Wolf's and Barth's critique against the particularism of the first ethnographies (namely, Boas' or Evans-Pritchard's theories). These first anthropologists maintained an isolated and separated conception of culture and consequently, they were unable to apprehend links between different human groups. In contrast, I will mention new approaches (transnationalism and the anthropology of the boundaries), more sensible to the differential situations in borders and the existence of transnational nets among regions. Lastly, I will allude to the difficulties that three contemporary anthropologists (Appadurai, García Canclini and Kearney) must face, when they link these new approaches with some postmodern concepts (such as "rhizome", "porous border" or "hybridity") and therefore, they cannot apprehend either the complexity of the boundaries.
\end{abstract}

Key words: boundary, postmodernity, theoretical frame, particularism, transnationalism

\section{Resumen}

En este artículo se va a tratar de comprender cómo la elección del marco teórico va a tener consecuencias en la realidad estudiada, en el caso concreto de la antropología de los espacios fronterizos. En primer lugar, nos referiremos a las críticas de Wolf y Barth al particularismo característico de las primeras etnografías (como las de Boas y Evans-Pritchard), dado su concepto aislado y cerrado de cultura, que les impedía reconocer los vínculos y contactos entre distintos grupos humanos. Como contraste a los particularismos, recientemente han aparecido nuevos enfoques teóricos, más atentos a las realidades fronterizas y al establecimiento de redes transnacionales entre distintas regiones; entre ellos, destacaremos las aportaciones de tres antropólogos contemporáneos (Appadurai, García Canclini y Kearney) quienes vinculan los enfoques transnacionales con algunos conceptos propios de la filosofía posmoderna (como "rizoma", "frontera porosa" o "hibridez") y como consecuencia tienen dificultades aprehender la complejidad de los espacios fronterizos.

Palabras clave: frontera, posmodernidad, marco teórico, particularismo, transnacionalismo.

\section{Declaración de motivos, aclaraciones conceptuales y genealogía del problema tratado}

El interés que guía esta investigación es tratar de comprender la influencia que una corriente teórica muy en boga como la posmodernidad puede tener en una disciplina empírica y con vocación comprensiva como la antropología, en el caso concreto del estudio de los espacios fronterizos. Sin embargo, el principal objeto de estudio de este 
artículo no será tanto la realidad estudiada por los antropólogos, sino las teorías o marcos teóricos de los que parten, en lo que podríamos denominar "epistemología de la antropología". Cabe mencionar una aclaración conceptual que puede resultar de utilidad para ubicar este escrito: siguiendo a Popper, mantendremos que "no hay hechos sino para teorías" (1962:49), es decir que al optar por un marco teórico determinado (por ejemplo un enfoque moderno o posmoderno) la misma realidad estudiada (en este caso, la frontera), va a ser entendida de modo distinto. Ahora bien, esta constatación no va a suponer en ningún momento una postura escéptica 0 relativista (entendiendo por el primer adjetivo, la posición que duda de la posibilidad del conocimiento de la realidad; y por el segundo, la convicción indiferente de que un marco teórico es igual que otro, no siendo posible comparar distintas aproximaciones teóricas y decidirse por una de ellas). En contraste con estas actitudes, desde un primer momento admitimos partir de un enfoque realista epistemológico, que puede comparar y criticar distintos marcos conceptuales a partir de los cuales puede conocerse la realidad.

A continuación, nos referiremos a distintos momentos de la historia de la antropología (con ciertas confluencias de otras ciencias sociales), para situar el problema que pretende abordar este artículo. En primer lugar, cabe destacar la explicación de Moreno Feliú, quien entiende los primeros estudios de casos antropológicos como "la foto fija de la época clásica" (2010:50), que tendía a aislar las sociedades estudiadas en un marco espacial y temporal cerrado e invariante. Algunos ejemplos de esta primera aproximación teórica pueden ser las etnografías de Boas con los Innuits, los trabajos de Mead en Samoa y Nueva Guinea y los de Evans-Pritchard con los Azande y los Nuer.

La principal crítica que recibieron estas etnografías se debe al carácter monádico de sus conceptos que les impedían aprehender las relaciones y vínculos que se daban entre distintas formas de vida (o incluso, entre el antropólogo y la cultura estudiada). Es decir, al entender cada cultura como una unidad cerrada y claramente delimitada, estos primeros antropólogos no lograban aprehender los vínculos, relaciones y contactos que se establecen entre distintos grupos humanos.

Eric Wolf, cuya crítica epistemológica a las primeras etnografías inspira el presente artículo, fue uno de los primeros autores en poner en cuestión el modelo clásico y monádico de la antropología, y destacar, por el contrario, que la "humanidad constituye un total de procesos múltiples interconectados, y que los empeños por descomponer en sus partes esta totalidad [...] falsean esta realidad" (Wolf 1984:15).

Siguiendo esta crítica de Wolf, nos interesa destacar que los primeros antropólogos tomaban como punto de partida de sus etnografías una "unidad metodológica de indagación" (Wolf 1984:28) (el concepto cerrado y limitado de cultura al que ya nos hemos referido) y confundían esta unidad conceptual con la realidad estudiada, dando la impresión de que todas las culturas se encontraban separadas entre sí, obviando las interconexiones y contactos que se han dado a lo largo de toda la historia de la humanidad.

Puede resultar aclarador aludir a una crítica más contemporánea a esta confusión conceptual propia de las primeras manifestaciones de la antropología. Se trata del posicionamiento de Dolors Comas, para quien los primeros antropólogos entendían que cada cultura poseía "un conjunto de rasgos que le son propios, cuyos límites coinciden con los de un grupo humano y se concretan en una determinada área. Así, una cultura resulta ser específica y definible en el espacio y en el tiempo, siendo aquello que define e identifica a un grupo humano y lo diferencia del otro" (Comas 1998:31). A partir de esta comprensión localizada y bien determinada de las culturas, el trabajo de estos primeros antropólogos se entendía como "identificar y describir [sus] rasgos de forma más o menos detallada y exhaustiva" (Comas 1998:31).

Destacando el particularismo histórico y el individualismo metodológico de los estudios de casos, Comas también aduce que acababan manteniendo un punto de vista evolucionista que les impedía comprender tanto la diferencia cultural, como las relaciones que se establecen entre distintas formas de vida. Según esta autora, estas limitaciones conceptuales impedían a los primeros antropólogos comprender las conexiones que se dan entre distintas formas de vida. Además, en el momento en que se daban estudios comparativos entre culturas, "el punto de referencia compartido era la 'sociedad occidental' que se erige como el motor de los cambios y el punto de llegada de otras sociedades" (Comas 1998:35). Y como consecuencia de este enfoque, "el sentido lineal por el que se concibe la 
evolución hace que el resultado inevitable sea la uniformización cultural, a pesar de las variaciones locales que puedan existir" (Comas 1998:35).

Siguiendo con la genealogía de corrientes antropológicas y como contraste con los particularismos de la primera época, cabe destacar que a partir de los años setenta del pasado siglo, algunos antropólogos comenzaron a cambiar su enfoque teórico para tratar de aprehender las conexiones o contactos que se dan entre distintas formas de vida. El primero de ellos fue Fredrik Barth, quien cuestionó el primordialismo de la primera tradición antropológica para llevar a cabo otra aproximación al estudio de las culturas, que él denominaba "grupos étnicos". Este concepto implica que "cada cultura es un grupo discreto cuyos rasgos diferenciales se mantienen a lo largo del tiempo, aunque se extiendan a lo largo del espacio y entren en contacto con otros grupos" (Barth 1976:9). La novedad de este enfoque es que no parte de una concepción externa y predeterminada de cada cultura, entendida como un marco fijo, incuestionable y predefinido por el antropólogo que lo observa desde fuera; sino que, por el contario, se tiene en cuenta cómo los individuos que pertenecen a un grupo se "identifican a sí mismos y son identificados por otros" (Barth 1976:11), para tratar de comprender la distribución de distintos grupos étnicos y sus relaciones.

Ahora bien, en el momento de transición entre paradigmas que cabe situar la obra de Barth, debemos destacar que aunque se mostrara interesado por los vínculos y tensiones que se establecen entre distintos grupos, sigue entendiendo a cada cultura como una comunidad cerrada y bien delimitada, generalmente por fronteras estatales. Es decir, pese a reconocer, por ejemplo que pastunes y baluches suelen establecer relaciones comerciales, en ningún momento se plantea que estos dos grupos puedan cambiar su localización o que puedan establecerse relaciones interétnicas.

Un poco más adelante en el tiempo, estas claras delimitaciones étnicas y fronterizas van a ser progresivamente superadas, tanto por el reconocimiento (ya anticipado por Wolf y Barth, entre otros) de que a lo largo de la historia de la humanidad siempre se han dado vínculos y contactos entre distintos grupos humanos; como por la aparición de nuevos enfoques teóricos interesados más por los vínculos y movimientos de grupos humanos, que por los compartimentos estanco, como por ejemplo, las primeras etnografías de la frontera. Destaca en este sentido la investigación de David Newman y Anssi Paasi, quienes en nombre de la ya mencionada crítica al particularismo histórico y al nacionalismo metodológico, orientan su investigación a la vida en la frontera y distinguen diferentes factores para el análisis de este espacio, concediendo una atención especial a las relaciones de inclusión/exclusión que se establecen en la frontera y que crean una identidad fronteriza, distinta a la identidad de los que viven en el interior de cada territorio. En sus palabras: "En antropología, nuevos enfoques han desafiado los supuestos acerca del isomorfismo del espacio, los lugares y la cultura, enfatizando la necesidad de evaluar los roles simbólicos de los límites estatales y fronteras" (1998:194).

Debemos tener en cuenta, además, otro enfoque teórico que se interesa por los contactos y vínculos humanos más allá de los límites del estado nación, se trata de la perspectiva transnacional. Esta corriente teórica, iniciada en los años noventa, investiga "procesos y prácticas económicas, políticas y socioculturales que están vinculados a y configurados por las lógicas de más de un estado-nación, y que se caracterizan por el cruce constante de sus fronteras" (Suárez 2008:991). Este nuevo enfoque no dirige su atención a los grupos humanos concentrados en un espacio o región concreta, sino que estudia la extensión de estos grupos entre distintos países, con especial interés en aprehender los vínculos y redes que establecen estos grupos y superan las fronteras nacionales. Podemos destacar distintos ejemplos de esta corriente, como las etnografías de procesos migratorios realizadas por Levitt, De Wind y Vertovec y por Glick Schiller, Blash y Blanc-Szanton, así como los estudios de redes migratorias aplicados a la realidad española de Aparicio y Tornos, y distintos análisis de movimientos diaspóricos globales, como los estudios de Clifford y Sorensen.

Sin embargo, y alcanzamos aquí el principal objeto de estudio de este artículo, lo que en un primer momento suponían novedosos enfoques teóricos, adecuados para investigar una realidad concreta (la vida en la frontera o los flujos migratorios), comienzan a extenderse y generalizarse, para convertirse en el marco teórico privilegiado para estudiar cualquier tipo de realidad. Además debemos destacar, por ejemplo siguiendo a Paasi, que estos 
enfoques comenzaron a recibir influencia de la filosofía posmoderna, especialmente de la obra de Deleuze y su concepto de rizoma, para iniciar una serie de investigaciones, que podemos denominar en un primer momento "transversales" o "fluidas", si seguimos la terminología de Bauman, para proponer una serie de teorías que Paasi no duda en denominar "crípticas" (2010:17).

En este momento puede resultar aclarador otro matiz conceptual: entendemos por "posmodernidad" aquella corriente de pensamiento iniciada en Francia en los años setenta del pasado siglo, caracterizada por la subversión (en ocasiones también denominada "inversión" o "deconstrucción") de las categorías utilizadas tradicionalmente por la filosofía moderna (como "categoría", "sistema" o "jerarquía") para proponer una comprensión de la realidad "rizomática", esto es, asistemática y sin jerarquías, donde prima el caos y la confusión vital, antes que la claridad y distinción del orden teórico. Por concretar, cabe caracterizar a esta corriente con uno de sus lemas fundadores, el título del ensayo de Berman de 1982: All that is solid melts into air.

Algunos ejemplos de esta vinculación de perspectivas pueden ser la "geografía posmoderna" de Edward W. Soja, según la cual, los límites entre las naciones han quedado difuminados por las conexiones transnacionales. Este marco es usado también en estudios feministas, por ejemplo en las obras de Bell Hooks y Cherry Moraga, para quienes la realidad transfronteriza queda vinculada con el cuerpo de las mujeres, especialmente mestizas y chicanas, entendidas como una frontera (o puente) indescifrable y transido por múltiples diferencias.

Una vez presentada la genealogía y el presente de este marco teórico, en la segunda parte de este artículo explicaremos la obra de tres autores que parten de la perspectiva transnacional y reciben influencia de la posmodernidad, para proponer una antropología del espacio fronterizo, que presenta algunas dificultades, que serán descritas en la tercera parte de este artículo.

\section{Tres casos concretos de la antropología de la frontera, influida por la posmodernidad}

\subsection{Arjun Appadurai y los paisajes de la diáspora}

El primero de estos autores donde confluyen el interés por la perspectiva transnacional y la posmodernidad es el antropólogo hindú Arjun Appadurai, quien inicia su obra Modernity at large (1996) cuestionando la clara delimitación tanto de fronteras, como de grupos humanos, propia de las primeras corrientes antropológicas que ya hemos descrito. Aunque este autor reconoce que los flujos migratorios han existido en toda la historia de la humanidad, insiste en que a lo largo del siglo XX se han incrementado, tanto en cantidad, como en velocidad. La consecuencia más destacada de este cambio es la aparición de un nuevo orden de inestabilidad: disminuye el poder de los estados naciones y el espacio se va desterritorializando, de modo tal que ya no resulta posible identificar imágenes culturales con contextos locales, sino que los imaginarios se hacen fluidos y se dispersan y extienden a lo largo del planeta.

Este autor define un nuevo modo de migración, que denomina "diáspora" (1996:10) y que se encuentra profundamente vinculada con el incremento de la imaginación. Según Appadurai, gracias a la expansión y a la fuerza de los medios de comunicación y de las nuevas tecnologías (como Internet), cualquier sujeto, de cualquier parte del mundo, puede conocer otras realidades y desear acceder a un mundo mejor, lo que le impulsa a querer cambiar sus circunstancias e incluso a migrar. Estos nuevos vínculos transnacionales (en un primer momento, imaginarios o digitales; pero con consecuencias en la realidad, como el fomento de las migraciones) empezaron a proliferar a partir de la década de los ochenta, y han cambiado la comprensión del mundo, al romper las distancias y las barreras características, según él, de la modernidad. Distingue de este modo Appadurai un nuevo espacio global que denomina "rizomático" (1996:29), utilizando la expresión de Deleuze y que se caracteriza por la desterritorialización del espacio, la heterogeneidad cultural y el transnacionalismo.

Ante las insuficiencias de las categorías tradicionales del pensamiento, entre las que incluye la antropología de casos, mencionada en las primeras páginas de este artículo; el marxismo, así como los modelos que distinguen entre centro y periferia, como el sistema mundo de Wallerstein y el modelo de atracción o expulsión [push-pull 
models] básico en el estudio de migraciones, este autor acuña un nuevo concepto o marco de estudio para analizar estos flujos globales.

Se trata del "paisaje" [scape], nueva categoría de análisis, adecuada a esta nueva realidad cambiante y dispersa. El concepto es un juego de palabras de gran riqueza semántica y muy interesante para este trabajo, pero de difícil traducción. El término escape significa "huida" o "dispersión", pero el sufijo scape, en palabras como landscape, designa un espacio concreto, en este caso, un paisaje. Los escapes de Appadurai referirían por lo tanto a algo así como un "espacio en movimiento continuo".

Estos paisajes o escapes se dividen en cinco subcategorías, adecuadas a distintos ámbitos de estudio. Se trata de los ethnoescapes (referidos a la antropología y la diáspora), los mediaescapes (referidos a los flujos de información), los tecnoescapes (referidos a las nuevas tecnologías y la informática), los financescapes (referidos a flujos económicos) y por último los ideoescapes (referidos a las imaginación global, así como las distintas ideologías políticas extendidas por el mundo) (1996:35).

Para centrarnos en el tema de este trabajo, nos vamos a ocupar del concepto de "etnopaisajes" (ethnoescapes), que el autor propone para definir una antropología transnacional ocupada del estudio de los flujos globales y cosmopolitas que superan los "grandes relatos modernos" (1996:48), en una nueva comprensión del espacio "doblemente borrosa" (1996:50, el concepto original es post-blur-blur), donde una multiplicidad de narrativas subversivas, críticas y cambiantes, altera lo antes era una comprensión clara y delimitada de la realidad.

También resulta interesante observar cómo estos cambios producidos en la posmodernidad afectan, según Appadurai, a la configuración de los Estados mismos, que en las últimas décadas del siglo XX se han convertido en diaspóricos y cambiantes, por la profusión de personas (alude literalmente a "refugiados, turistas, trabajadores, invitados, ilegales e intelectuales" 1996:160) que traspasan sus fronteras, así como por el aumento de la imaginación que lleva a pensar otras formas de configurar el Estado. De este modo, la comprensión clásica del Estado como un todo cerrado con fronteras claramente delimitadas debe ser superada, para aportar un nuevo concepto de Estado fluido y cambiante, fragmentado y disperso. Ahora bien, esta nueva concepción no es siempre positiva, ya que según este antropólogo, los conflictos pueden aumentar con este desdibujamiento de las fronteras, ya que van a seguir existiendo minorías perseguidas y surgirán nuevos movimientos étnicos y nacionalistas, extendidos ahora de modo transnacional y reforzados por la debilitación del estado.

Todo este análisis lleva al autor a postular un nuevo espacio global y confuso: un "mundo desterritorializado, diaspórico y transnacional" (1996:188) donde se da una tensión constante entre los Estados "modernos y tradicionales" y los flujos de población que tratan de atravesarlos y superarlos. Esta ecuación se hace más compleja cuando estos flujos y tensiones no sólo se dan en el espacio fronterizo, de modo físico; sino que se extienden gracias a los medios de comunicación y las nuevas tecnologías de la información, que aportan nuevas formas de mediación, fomentan la imaginación social y reproducen las críticas al estado tradicional. De este modo, Appadurai concluye su análisis destacando la fragilidad de esta nueva configuración del espacio, tanto por la aparición constante de nuevos e inesperados vínculos entre regiones o comunidades antes remotas y separadas, como por el surgimiento de nuevos espacios, fragmentados y cambiantes. Insiste asimismo en que esta realidad rizomática, diaspórica y subversiva supone un desafío, tanto para el teórico que trata de aprehenderla, como el Estado Nación que trata de frenarla; en la apertura de un proceso dialéctico y subversivo interminable.

\section{2 (Viva) Tijuana híbrida en la obra de García Canclini}

En segundo lugar, nos referiremos a la obra del antropólogo mexicano Néstor García Canclini, quien parte de un enfoque transnacional y posmoderno, interesado (en principio) en las corrientes artísticas que se dan en un espacio concreto: la frontera que separa México de EEUU. Al igual que Appadurai, este autor también inicia su teoría en la crítica al particularismo que aludimos al inicio de este escrito, para sostener con marcado acento posmoderno que "las culturas resultan imposibles de delimitar" (2001:36). El concepto básico de este antropólogo es el de "hibridación", que cabe entender como la alteración de las categorías utilizadas en la modernidad para organizar y 
clasificar los sistemas culturales, entre las cuales destacan las "parejas organizadoras de las ciencias sociales: identidad/diferencia, local/global, tradición/modernidad o norte/sur" (2001:13).

Según García Canclini, la posmodernidad no debe ser entendida como una nueva etapa en la historia, sino como un "modo de problematizar los conceptos básicos de la modernidad y mostrar sus deficiencias conceptuales a la hora de comprender fenómenos complejos con la vida en la frontera" (2001:44). Entonces, ciertas nociones básicas de la modernidad, como emancipación, cultura, renovación y democracia siguen vigentes en el presente, pero son redefinidas y problematizadas por el enfoque posmoderno, que trata de aprehender las realidades híbridas que pasaban desapercibidas para las “teorías modernas" (2001:51).

Ahora bien, esta modificación de los modelos clásicos no se da sólo a un nivel teórico (podríamos decir "categorial") sino que según el autor llega a tener consecuencias en el espacio, como por ejemplo la desterritorialización. Cabe definir este concepto como la pérdida de la relación (adjetivada por García Canclini como "natural" 2001:281) que se establecía tradicionalmente entre un espacio geográfico y una cultura concreta. Esta clara delimitación, característica de la modernidad, resulta imposible en el presente, cuando aumenta el tránsito de flujos migratorios y el fomento de las nuevas tecnologías provoca la relativización de las diferencias culturales y la extensión global de unos mismos símbolos y unos mismos géneros, denominados "impuros" (2001:282) por el autor.

Esta nueva comprensión híbrida e impura de las realidades humanas contrasta con la modernidad, que el autor caracteriza (en este momento y pese a lo mantenido anteriormente) como una época distinta "donde las poblaciones eran homogéneas y cerradas" (2001:260) y se establecían claras diferencias entre centro y periferia, o norte y sur; diferencias creadas y mantenidas por una "red de poder creada por las potencias coloniales" (2001:282).

Sin negar la superación o eliminación de estas jerarquías, que persisten en nuestros días, para García Canclini el panorama internacional contemporáneo se ha vuelto más difuso y complejo, dada la profusión de flujos migratorios y movimientos interculturales que esparcen la diferencia por el mundo y problematizan el esquema dual y vertical de poder, clásico de la modernidad. Destaca también la profusión de culturas híbridas, e inclasificables por los esquemas tradicionales, como la de Tijuana, "que inauguran una nueva concepción del espacio y de la existencia" (2001:283), que el autor describe con una cita de Certeau: "La vida consiste en pasar constantemente de fronteras" (2001:286).

Aunque en cierto momento García Canclini reconoce que la hibridación cultural ha existido siempre, al menos en Latinoamérica desde el "primer sincretismo entre España, Portugal y las culturas indígenas" (2001:297), insiste en que este fenómeno se ha radicalizado en el presente, con la aparición de "nuevos vínculos culturales, relaciones sociales, y de nuevos conflictos, que ya no suponen el enfrentamiento solo de grupos radicalmente opuestos, sino que se multiplican los frentes, se dividen las reivindicaciones y aumentan los problemas de modo exponencial" (2001:315). Todo esto le lleva a sostener que todas las culturas son híbridas y fronterizas, y que sus relaciones se multiplican en un "único territorio global, plagado de tensiones y de diferencias, donde nuevos actores intentan subvertir y deslocalizar las relaciones de poder" (2001:316).

\subsection{El final de la frontera, según Michael Kearney}

En tercer y último lugar, aludiremos a un artículo del antropólogo estadounidense Michael Kearney, Fronteras y límites del Estado y el Yo al final del imperio, donde el enfoque posmoderno es referido para destacar las dificultades de una frontera concreta: la que separa México de EEUU, que queda definida como un espacio fluido y poroso, constantemente traspasado.

Distingue este autor dos momentos en la historia de esa frontera. En primer lugar, en lo que él denomina la época del Estado Nación, los límites (tanto entre fronteras, como entre identidades) estaban claramente definidos y eran, según Kearney, "absolutos" (2003:48), momento correspondiente a la situación colonial, donde existía una clara 
diferenciación espacial, categórica e identitaria entre México y EEUU (en sus palabras: "el anglo era estadounidense y el mexicano era el otro" 2003:49). Sin embargo, en un momento posterior, denominado por Kearney "transnacional" o "posnacional" (2003:49) a raíz del proceso de descolonización se da el "colapso de la distinción categórica entre el yo imperial y el otro colonial" (2003:58), por lo que ya no resulta posible mantener tales límites, ni entre identidades, ni entre fronteras y todo se confunde, se vuelve ambiguo y poroso. Literalmente, según Kearney, las antes claras fronteras de EEUU, se encuentran amenazadas por "miles de indocumentados [que] desafían con éxito el poder del Estado para controlar su movimiento de entrada a su espacio" (2003:52). Este autor encuentra que, según la etnografía de la migración transnacional, las "comunidades están constituidas transnacionalmente y, entonces, desafían el poder que define a los estados-nación que trascienden" (2003:53) y que incluso los amenazan, puesto que el "asalto a la integridad de sus límites [del Estado-nación] es un asalto a su poder" (2003:60). Como efecto de tal colapso y de tal confusión generalizada, el autor insiste en que este proceso puede llevar a la "declinación del imperio estadounidense", nación que está experimentando, en la actualidad y según Kearney, una "disolución espacial y simbólica entre él y sus colonias" (2003:52).

En este modelo transnacional la frontera adquiere, siempre según Kearney, una naturaleza indeterminada, imposible de categorizar con los conceptos tradicionales. En sus palabras: "En el área fronteriza esta distinción [yo/otro] una vez espacial, categórica y muy política, es cada día más borrosa" (2003:61). Además, la frontera ya no sólo se ubica en el límite entre EEUU y México, sino que todo el país se hace zona fronteriza. Define entonces el autor este espacio fronterizo como: "una línea sin anchura [...] una zona social y cultural de amplitud indeterminada y se podría decir que corre profundamente desde México hasta Canadá" (2003:55).

Kearney acaba su artículo con la descripción de un apocalíptico panorama fronterizo donde la "configuración cambiante desafía la habilidad de los Estados [...] para definir las identidades legales y culturales de sus poblaciones fronterizas" y de este modo se dan, según este autor, "dos formas de decadencia" (2003:61): la de los Estadosnaciones tradicionales, que no pueden hacerse cargo de los indocumentados que constantemente penetran en su territorio; y la de la antropología, como disciplina teórica, que víctima de la "crisis de la representación" característica de la posmodernidad, persiste en la utilización de unas categorías que ya no resultan válidas para describir esta realidad porosa y cambiante.

\section{Principales críticas a estas teorías}

Cabe comenzar este apartado destacando que en un primer momento estos tres autores se refieren a un rasgo innegable de nuestros días: el aumento de flujos, tanto humanos, como de capital, que superan los límites del Estado Nación (tanto por aparición de grandes corporaciones y empresas multinacionales que superan estos límites; como por la profusión de nuevas tecnologías, tanto de la información, como del transporte, que facilitan la movilidad de las personas y el contacto entre regiones que antes se encontraban más separadas, cabría decir, desconectadas). Es decir, no resulta posible negar el hecho de la globalización en el presente. Ahora bien, al insistir y centrarse en este fenómeno e identificarlo como una manifestación de una nueva época, la posmoderna, estos autores se exceden en sus análisis de dos formas bastante problemáticas, que pasamos a describir.

En primer lugar, los tres han idealizado o romantizado el pasado, en la proyección de una imagen idílica e inmovilista (similar a la proyectada por los estudios de casos), según la cual, tanto las fronteras entre regiones, como las identidades grupales, se encontraban claramente delimitadas en la modernidad, sin que se prestaran a confusión, ni a mezclas. Este punto de vista se aprecia con claridad en la concepción de Kearney de los límites "absolutos" que separaron México y EEUU en la época colonial; pero también aparece en la obra de los otros dos autores, tanto en la ambigüedad del estudio de García Canclini, que no llega a definir si la posmodernidad es una nueva etapa en la historia o una nueva forma de análisis, distinta a la modernidad; como en la de Appadurai, quien destaca el incremento de flujos de población en el presente, por efecto de los nuevos medios, obviando por ejemplo todas las diásporas sucedidas en la historia de la humanidad.

Como contraste a esta (no tan obvia) separación de espacios y de identidades en la modernidad y como un modo de responder a las limitaciones de las primeras etnografías, estos autores mantienen que en el presente estos 
límites se han desdibujado, de tal modo que ya no es posible localizar fronteras claras, sino que todo está mezclado, confuso, híbrido o poroso y es prácticamente imposible de aprehender o describir. Ahora bien, en oposición con esta clara y estricta diferenciación de etapas históricas que caracteriza a las propuestas de estos autores, podemos mencionar cómo los límites históricos entre fronteras han estado disputados a lo largo de la historia (piénsese, en las pugnas entre Francia y Alemania, por Alsacia y Lorena, en el proceso colonial mismo o en la conquista del Oeste) y aunque la existencia física de algunas fronteras (como los Pirineos, el estrecho de Gibraltar o el río Bravo) resulta incuestionable, nunca ha dejado de darse un tránsito a través de estos territorios, en constante tensión con las políticas de control de los distintos estados.

En este sentido y en oposición a esta idealización, cabe recordar con Liliana Suárez que siempre ha habido tránsito de población entre fronteras, así como redes transnacionales. En sus palabras: "las redes y conexiones establecidas por los migrantes entre origen y destino son tan antiguas como los procesos migratorios en sí mismos, y la historia lo ha demostrado contundentemente" (2008:918). Alude entonces esta autora a los distintos procesos diaspóricos, así como a migraciones transoceánicas en el XIX y continúa su crítica sosteniendo que "el transnacionalismo precede a la nación" (2008:919).

El segundo exceso que cometen estos autores es la generalización o totalización de sus análisis. De nuevo, en palabras de García Canclini: "todas las culturas son híbridas y fronterizas" (2001:316) o como también ha sostenido Kearney "se podría decir que [la frontera] corre profundamente desde México hasta Canadá" (2003:55) o en la expresión literal de Appadurai: todo el planeta se ha convertido en "mundo desterritorializado, diaspórico y transnacional" (1996:188).

Siguiendo la crítica de Suárez, cabe destacar cómo dada la extensiva y exhaustiva aplicación del marco teórico del transnacionalismo para estudiar distintas realidades sociales, este concepto va perdiendo su potencial heurístico, analítico y su capacidad crítica, para convertirse en un "adjetivo vacío, sin ninguna utilidad analítica" (2008:792). Por ejemplo, la etnografía de García Canclini en Tijuana resulta de gran interés y nos puede ayudar a comprender la complejidad de este espacio fronterizo; pero de este estudio no cabe concluir que todos los espacios sean igualmente fronterizos, híbridos o problemáticos y por ello indiscernibles.

Como también ha criticado Paasi (2010), estos enfoques posmodernos acaban dando lugar a unos análisis bastantes confusos, donde resulta prácticamente imposible delimitar el espacio estudiado. Dado este exceso de abstracción y como contraste a las claras delimitaciones que se establecían en los estudios de casos, en este momento y bajo esta nueva óptica, ciertos autores (como Kearney) insisten en que las fronteras han desaparecido; de este modo, los flujos humanos en constante movimiento y dispersión se convierten en el principal (aunque en ocasiones indiscernible) objeto de estudio de estas disciplinas posmodernas. Por otro lado, continúa su crítica Paasi, también por influencia de la posmodernidad, otros autores (como García Canclini) van a mantener que toda realidad espacial es fronteriza. $Y$ en ninguno de estos casos se entiende nada, ya que unos insisten en que no existe la frontera y otros en que todo es frontera y en ambos, han perdido de vista las dificultades reales que se dan en este espacio concreto. Es decir, cuando se extienden y generalizan estos conceptos posmodernos, la frontera se hace ininteligible y el estudio de este espacio, impracticable.

Es decir, estos tres autores han tratado de explicar los cambios sucedidos en una nueva época, creando nuevos conceptos, adecuados a lo que ellos entienden como una realidad inabarcable, móvil y compleja; pero, desorientados por esta nueva concepción del presente y acusando exceso de influencia de la posmodernidad, acaban proponiendo una serie de conceptos abstractos y confusos, a partir de los cuales resulta muy difícil aprehender la realidad.

Siguiendo esta crítica por exceso de generalización que pierde de vista el objeto estudiado, podríamos decir que los tres casos presentados adolecen de atención al detalle, a las diferencias de cada espacio fronterizo concreto o a la trayectoria vital de cada sujeto que trata de cruzarla. Tampoco han tenido en cuenta estos autores los distintos factores e intereses que pueden impulsar a alguien a tratar de traspasar una frontera. Es decir, tanto García Canclini, como Appadurai se refieren (con bastante optimismo) a la desaparición de las fronteras y el aumento de 
flujos migratorios (por la creación cultural, híbrida y novedosa, en el caso del antropólogo mexicano y por el aumento de imaginarios y de contactos virtuales, gracias a los nuevos medios, en el caso del antropólogo indio); pero ninguno de estos dos autores tiene en cuenta los factores económicos que subyacen a estos movimientos transnacionales (ni que, dicho de modo un tanto burdo, no todo el mundo puede disfrutar de la riqueza cultural de Tijuana, ni tener acceso a una conexión a Internet que permita imaginar una nueva vida). Por su parte, Kearney se muestra preocupado (con bastante pesimismo) sobre el aumento de flujos migratorios que van a causar la desaparición de una supuesta identidad estadounidense, y no tiene en cuenta cómo el proceso de formación identitaria de este país se debió, en mayor medida, a la llegada de distintas olas de migración; ni tampoco contempla cómo la economía estadounidense se ve beneficiada en la actualidad, por los grupos de migrantes procedentes de Latinoamérica.

Podemos destacar en este momento la principal crítica de este artículo a estos enfoques posmodernos, que confunden el marco teórico elegido (la perspectiva transnacional, la hibridez, el rizoma o el concepto poroso de frontera) con la realidad estudiada; y persuadidos de que la realidad debe corresponderse a estos marcos, pasan desapercibidos o quedan desdibujados elementos muy relevantes para aprehender la complejidad de cada espacio fronterizo.

De modo paralelo a la crítica de Wolf a los antropólogos clásicos por confundir conceptos (claros y bien delimitados) con la realidad, que daba lugar a una comprensión atomista de las distintas culturas, como bloques homogéneos sin contacto entre sí; cabe decir que estos tres autores han confundido conceptos posmodernos (difusos o porosos) con la realidad estudiada, atribuyéndole este carácter rizomático, fluido o híbrido, imposible de aprehender (cuando en realidad, lo que resulta difícil de aprehender no es la realidad estudiada, sino los conceptos, como sucede con "rizoma").

Es decir, como respuesta a las limitaciones conceptuales de los estudios de casos y del particularismo, las nuevas corrientes antropológicas, influidas por la posmodernidad, han definido el espacio de forma fluida, cambiante, y han centrado sus estudios en los flujos trasnacionales, movimientos diaspóricos y momentos de hibridez, superando u obviando realidades como las fronterizas. Pero al confundir la categoría o concepto usado (borrosidad de fronteras, diásporas o cultura híbrida) con la realidad; llegan a sostener que la frontera es borrosa, cambiante, inasible, e incluso fácilmente superable.

Y del mismo modo que, si al incidir en los límites claros entre fronteras, se obvian las interconexiones y vínculos que se establecen entre distintas culturas; en este segundo caso, al incidir en dimensiones como la fluidez de los tránsitos transnacionales o la porosidad (cuando no desaparición) de las fronteras, se obvian o anulan los problemas de todos aquellos que pretenden cruzarla e incluso pierden la vida tratando de superarla. En este momento y como contraste con estos enfoques porosos y fluidos, cabe citar los estudios de Wayne A. Cornelius (2001), quien muestra cómo el aumento del control en la frontera entre México y EEUU ha provocado un incremento en el fallecimiento de migrantes que tratan de cruzarla, que oscilaba, en el inicio del nuevo siglo en quinientas víctimas al año.

Conviene recordar asimismo con Dietz la necesidad de no caer en extremos, es decir, la superación crítica de enfoques particularistas no debe conllevar la adopción de enfoques arbitrarios y relativistas, que en nombre de la posmodernidad incidan en el carácter construido y artificial de todos los conceptos (y por extensión, de todas las realidades) como "si la invención de tradiciones y la imaginación de comunidades estuvieran regidas por un simple todo vale" (Dietz 2003:89). Ya que hay realidades menos construidas que otras y vidas en juego, por ejemplo a la hora de cruzar fronteras.

Cabe destacar asimismo cómo es posible descubrir un eje de poder-saber en esta conceptualización posmoderna de la frontera. Como argumentó Foucault, en el inicio de la Edad Moderna (para él, siglo XIX) las antiguas formas de poder y de control se suavizan y recubren "por la administración de los cuerpos y la gestión calculadora de la vida", en lo que considera el inicio del biopoder. Destaca el filósofo francés que en esa época aparecen nuevas instituciones ordenadoras de los cuerpos (escuelas, colegios, cuarteles y talleres), al mismo tiempo que surge un 
nuevo tipo de saber que favorece a los poderes establecidos y oculta las realidades que no le convienen. Entre estas nuevas disciplinas el autor destaca "prácticas políticas y observaciones económicas, problemas de natalidad, longevidad, salud pública, vivienda y migración" (2003:148).

Siguiendo este análisis del filósofo francés, cabe aducir que la mayoría de autores que anuncian el final de la frontera (como Kearney), consideran fácil la superación de este espacio (como Appadurai) o celebran la diversidad y riqueza de estos lugares (como García Canclini) y escriben desde lo que podríamos denominar "la parte privilegiada o poderosa del eje de saber-poder". Es decir, estos autores teorizan en consonancia con una serie de poderes e instituciones (académicas y estatales) que se apoyan mutuamente para definir una imagen de la realidad concreta (en este caso, una frontera difusa, fácilmente traspasable) que obvia las circunstancias y dificultades de los migrantes que tratan de cruzarla desde "el otro lado". Aunque, de hecho, resulta más sencillo anunciar el final de las fronteras cuando no se encuentran dificultades para cruzarla (sea por poseer los documentos necesarios para el control de aduanas o por una situación socioeconómica estable que permita costear los gastos del traslado). Por lo tanto, y ante esta nueva confusión entre teoría y hechos, constatamos que las fronteras siguen existiendo como una realidad problemática y situada, pese al énfasis posmoderno en su disolución o en su extensión ilimitada.

Cabe resumir la principal dificultad de los enfoques posmodernos que hemos descrito en este trabajo con una acertada crítica de Terry Eagleton, quien destaca el carácter ambivalente de los posicionamientos posmodernos en relación con la totalidad. Según este autor, "en una interesante ambivalencia puede descubrirse a algunos radicales que denuncian la totalidad a la que toman como lo real y a otros que renuncian a todo como una ficción de un cerebro recalentado y compulsivamente totalizador. Algunos [...] asumirán que el sistema hegemónico es completamente negativo -que nada en este todo no contradictorio y sin fisuras puede ser de valor, por definicióny pasarán de esta decadencia a idealizar a cierto otro numinoso" (Eagleton 1997:24-25). La consecuencia de este rechazo de todo lo presente y lo real, en nombre de lo completamente otro es, según este crítico, que este pensamiento "será en seguida preciosamente utópico, lanzando su cabeza contra los límites del lenguaje para poder vislumbrar cierto estado generalmente inconcebible más allá de él y un fantástico desplazamiento de un punto muerto genuinamente político" (1997:24).

El posmodernismo, sostiene el autor inglés, "puede ser casi tan exclusivista y censor como las ortodoxias a las que se opone" (1997:51). Es decir, aunque la gran mayoría de autores posmodernos escriban en nombre de la alteridad o la diferencia, con un marcado carácter relativista, en realidad este movimiento "está pleno de prescripciones morales universales, tales como la hibridez es preferible a la pureza; la pluralidad a la singularidad; la diferencia a la auto identidad" (1997:54), de tal modo que imponen su marco teórico (híbrido y diferencial) sobre la realidad. Destaca este pensador inglés una paradoja a la que se enfrentan la gran mayoría de estos autores posmodernos que "en sus escarceos con el antirrealismo, tienden a negar la posibilidad de describir cómo son las cosas" (1997:54), pero acaban argumentando e imponiendo de forma dogmática y totalitaria cómo es o tiene que ser el mundo.

Traduciendo esta crítica a nuestro ejemplo, cabe destacar que en el intento de criticar la clara delimitación de fronteras de los estudios clásicos, estos autores llegan a imponer y generalizar que las fronteras son (y tienen que ser) fluidas, porosas y rizomáticas; y como consecuencia pierden de vista (de modo, podríamos decir "miope") la realidad, plural y compleja, de los espacios fronterizos.

\section{Conclusiones}

Para acabar esta reflexión sobre la mutua influencia de marco teórico elegido y realidad estudiada, queremos destacar que esta crítica al enfoque posmoderno en antropología no implica un rechazo frontal a la perspectiva transnacional, ni siquiera a algunos enfoques novedosos, definidos por la posmodernidad, para proponer la regresión a un momento anterior del pensamiento, correspondiente quizás al estudio de casos. En un sentido muy distinto y aceptando que no se puede frenar el curso de los tiempos. La principal intención de este artículo ha sido destacar cómo al partir de distintos enfoques teóricos, se podía cambiar la percepción de la realidad estudiada. Ante esta constatación, se propone prestar más atención al método, ajustarlo para hacerlo más sensible a distintas 
realidades, en la búsqueda del equilibrio entre el marco teórico elegido y el objeto de estudio.

Sin mantener la clara y estricta separación de épocas, moderna y posmoderna, que afirmaban los autores criticados en este escrito, sí que admitimos que en el presente han surgido nuevos fenómenos o se han incrementado ciertas tendencias (por ejemplo, la aparición y extensión de nuevas tecnologías que permiten el establecimiento de vínculos transnacionales, con más intensidad y frecuencia que en el pasado), que exigen al teórico social un enfoque distinto, adecuado a estas nuevas realidades. En casos como éste, ciertos elementos o conceptos de la posmodernidad pueden resultar de interés para renovar las técnicas clásicas, pero se debe evitar siempre que el exceso de abstracción o teoreticismo impida aprehender el objeto de estudio, en su multiplicidad o diferencia. Cabe destacar en este sentido el desarrollo de distintas técnicas de investigación, cercanas a la posmodernidad, que resultan muy interesantes y enriquecedoras para estudiar los movimientos migratorios, como la etnografía multisituada de George E. Marcus, quien ante las insuficiencias de los estudios unilocales, que perdían de vista la conexión entre lugares de origen y de destino, propone un nuevo modelo de etnografía plurilocal, donde "no existe lo global en el contraste local-global tan frecuentemente evocado en estos tiempos. Lo global es una dimensión emergente en la discusión sobre la conexión entre lugares" (Marcus 2001:113). Y en esta nueva etnografía, el investigador "se encuentra con todo tipo de compromisos personales contradictorios. Estos conflictos se resuelven, tal vez de manera ambivalente, no al refugiarse en ser un antropólogo académico distanciado, sino en ser una especie de etnógrafoactivista, renegociando identidades en diferentes lugares" (2001:123).

Destacan asimismo otras técnicas, inspiradas en ciertos gestos de la posmodernidad, como la actitud del flâneur o paseante, quien sólo podía comprender la confusión de la ciudad moderna cuando caminaba. Esta idea ha servido a Monika Büscher, John Urry y Katian Witchger (2010) para diseñar técnicas móviles para la realización de etnografías, según la cual el antropólogo realiza, día a día, el mismo recorrido que la persona entrevistada, como un modo de inmersión, para conocer sus rutinas y el devenir mismo de su actividad cotidiana.

Ahora bien, seguimos insistiendo en que estas técnicas o modelos suponen unos marcos teóricos limitados, aplicables a realidades concretas, circunscritas espacial y temporalmente, y que no resultan generalizables, ni extrapolables de modo abstracto a toda la realidad, social o espacial. Cabe recordar en este sentido y para acabar, por ejemplo siguiendo a José Guilherme Magnani, que la antropología es un conocimiento situado en la realidad empírica, y que se a diferencia de otras disciplinas que presentan enfoques más totalizadores (él alude a la sociología, tras lo argumentado en este artículo podemos mencionar la filosofía), la antropología no se acerca a su objeto de estudio "desde fuera y desde lejos", sino "desde cerca y desde dentro" (Magnani 2002:12). Esto es, no de forma objetiva y externa, sino tratando de conocer a las personas, interesándose por sus problemas, hablando, observando y preguntando. Cabría decir, con los pies en el suelo antes que con la cabeza nublada por teorías y conceptos abstractos.

\section{Agradecimientos}

Este artículo no hubiera sido posible sin la paciencia, las recomendaciones de lectura y los buenos consejos de Jesús Sanz.

\section{Bibliografía}

Appadurai, A. 1996. Modernity at large. Minneapolis: University of Minnesota Press.

Barth, F. 1976. Los grupos étnicos y sus fronteras. México: FCE

Büscher, M; Urry, J. y Witchenger, K. 2010. Mobile methods. London: Routledge.

Comas, D. 1998. Antropología económica. Barcelona: Ariel 
Cornelius, W. 2001. Death at the border: efficacy and unintended consequences of US immigration control policy. Population and Development 27(4):661-685. http://www.jstor.org/stable/2695182

Dietz, G. 2003. Multiculturalismo, interculturalidad y educación: una aproximación antropológica. Granada: Universidad de Granada.

Eagleton, T. 1997. Las ilusiones del posmodernismo. Buenos Aires: Paidós.

Foucault, M. 2003. Historia de la sexualidad vol. 1. Madrid: Siglo XXI.

García Canclini, N. 2001. Culturas híbridas. Estrategias para entrar y salir de la modernidad. Buenos Aires: Paidós.

Kearney, M. 2003. Fronteras y límites del estado y el yo al final del imperio. Alteridades 13(25): 47-52.

http://www.uam-antropologia.net/pdfs/ceida/alte_25_6.pdf

Magnani, J. G. 2002. De perto e de dentro: notas para uma etnografia urbana. Revista Brasileira de Ciencias Sociais 17(49): 11-29. http://dx.doi.org/10.1590/S0102-69092002000200002

Marcus, G. E. 2001. Etnografía en/del sistema mundo. El surgimiento de la etnografía multilocal. Alteridades 11(22): 111-127. http://www.uam-antropologia.net/pdfs/ceida/alte_22_9.pdf

Moreno Feliú, P. 2010. Encrucijadas antropológicas. Madrid: Ramón Areces.

Newman, D. y Paasi, A. 1998. Fences and neighbours in the post-modern world: boundary narratives in political geography. Progress in Human Geography 22(2): 186-207. doi: 10.1191/030913298666039113

Paasi, A. 2010. A border theory: an unattainable dream or a realistic aim for border scholars? En: Wastl-Walter, D. The Ashgate Research Companion of Border Studies. Burlington: Ashgate, pp. 11-32.

Popper, K. 1962. La lógica de la investigación científica. Madrid: Tecnos.

Suárez, L. 2008. La perspectiva transnacional en los estudios migratorios. Génesis, derroteros y surcos metodológicos. En: García Roca, J. La inmigración en la sociedad española: una radiografía multidisciplinar. Barcelona: Bellaterra, pp. 911- 940.

Wolf, E. 1984. Europa y la gente sin historia. México: FCE.

Recibido el 15 Dic 2014

Aceptado el 4 Feb 2015 